\title{
Collecting and Visualizing Real-Time Urban Data through City Dashboards
}

\author{
STEVEN GRAY, OLIVER O'BRIEN and STEPHAN HÜGEL
}

\begin{abstract}
Dashboards which collect and display real-time streamed data from a variety of rudimentary sensors positioned in the built environment provide an immediate portal for decision-makers to get some sense of their city and environment. These devices are linked to previous renditions of control and management of real time services in cities, particularly transport, in control-room like setting but they are more flexible and do not require massive investment in hardware. At one level they are essentially simply screens linked to some sort of computational device whose displays are focused in web-page like formats. Here we catalog the experience of building such dashboards for large cities in Great Britain. In particular, we link these to the emergence of open data, particularly reflecting the experience of the London Data Store, and we then show how such dashboards can be configured in many different ways: as data tables which give some sort of physical presence to such data delivery, to purposes built dashboards for schools, and to various moveable displays that have artistic as well as informative merit. To an extent as real time streamed data becomes less of a novelty, we expect these dashboards to merge into more generic portals but for the moment that represents one very public face of the smart city and its big data.
\end{abstract}

\section{Introduction}

Dashboards have been providing system level operators with overviews of complex systems for many applications since they originated in the 1970's as "decision support tools and systems that served management, operations, and organizational planning" (Lafler, 2015). Mission Control type systems consisting of large displays, flashing lights, and streams of data filling multiple screens where operators pore over minute details of metrics from realtime systems have filled the imagination of children who have visited these control rooms or watched them in movie depictions of space missions or within headquarters of James Bond spy-like villains.

Yet when applying this to urban environments, cities and city managers have no equivalent systems to monitor the overall health of the city. Police and city officials have control rooms where operators can view live pictures from a vast numbers of CCTV cameras dotted around the city, for example. Central infrastructure such as Gas and Electrical networks such as the UK National Grid utilise general control room dashboards to monitor the supply and demand 
of these resources they provide, allowing them to match capacity in the supply chain when required, yet the general citizen has no idea about the real-time status of these systems.

Urban informatics has been defined as the use of data to better understand how cities work (CUSP, 2014). Of course this is little different from the practice of urban research and practice since urban planning became established institutionally over 100 years ago. Systematic and institutionalised planning has been based on notions first popularized by Patrick Geddes (1915) over one hundred years ago who argued that survey is essential to our understanding of cities, with data pertaining to the city as a prerequisite to effective planning and policy making. In fact, this generic definition of urban informatics as originating in data needs to be qualified. It is data that is digital that is of particular concern with the data being of many types from 'small' to 'big' in volume and scale, and from data generated in real time from sensors to data captured from individuals in their responses to social and economic functions.

In some senses, urban informatics is coincident with the smart cities movement which is focused on developing digital methods and tools for improving the performance of cities in terms of their efficiency and their equity (Batty and Hudson-Smith, 2005). Data is clearly a part of this in that it is the origin for the development of any intelligence that can be applied and embedded in the city, making it 'smart' or at least its citizens 'smarter'. There is nothing in this general notion of the smart city that is particularly digital but this terminology and our focus here adopts these ideas with respect to the digital world in general, and big data in particular.

Due to the comparative newness of these ideas, many of the tools that define urban informatics have multiple roles. There is a clear mandate to inform the way in which computation is being embedded in the city, In this sense, the tools that we introduce here which are all framed around the ideas of dashboards and data stores tend to produce information that provides a synoptic picture of what is happening in the city on a relative frequent basis, thus aiding understanding and providing fuel for defining urban problems. In short, because there are many different streams of data being generated in real time, and because we have only become aware of this during the past decade or so, the idea of dashboard as a convenient portal through which to view the real-time city has become an important way of focusing on what this new 'big' data can provide. Our purpose here is to explain, albeit briefly, what the prospect of the city dashboard opens up for our understanding and planning of the city.

\section{An Overview of CityDashboard}

CityDashboard which we show in Figure 1, is a data driven website that presents real-time metrics for various cities in the United Kingdom. Each live metric of the city is presented as a single widget on the main page of the website and is updated at various intervals without the need of for any refreshing. The platform was built as part of a JISC (Joint Information Systems Committee) funded research project in 2012 at the Centre for Advanced Spatial Analysis as a proof of concept that these sorts of systems could provide the general citizen with an overview of the short term 'daily' dynamics that a city might portray. 
The system consists of various services that collect data from open data platforms run by third parties and city data platforms. The system then normalises the data, archives and caches it to provide the users of the platform with an updated overview of the city without putting undue pressure on the underlying data sources that power the platform. The data is served to the interface in three different formats: a CSV file, a JSON object or an HTML widget. By serving the data to the interface in such a manner, we provide a rudimentary API (Applications Programming Interface) to serve the data to different platforms within the organisation for different research, development and/or applications projects. This allows other researchers to use the data collected to enhance their own research themes and ideas, a service which has been used successfully internally in the CASA lab where it was first deployed. The web interface uses the HTML view of the API as a first-class citizen of the backend service. This additionally serves as a check to make sure that the underlying collection is running, available and returning valid data from the external services.

The dashboard platform has been retrofitted to different cities within Great Britain. Currently the platform supports 8 cities around the country: Birmingham, Brighton, Cardiff, Edinburgh, Glasgow, Leeds, London, Manchester. Interestingly, London is by far the most active city on the platform. This is mainly due to the frequency and comprehensiveness of the live data streams available for London. Other cities on http://citydashboard.org serve as a tool for these cities to provide data to their residents and visitors, and to drive policy to open up data streams to the public. Due to the nature of the underlying platform, a new widget can be rapidly prototyped and added into the platform. If these datasets are available for multiple cities, for example Twitter trends within the cities, these widgets can be reused and made available to each of the the other 8 cities currently active with the only difference being that the data is spatially aggregated for that locale. The project has also repurposed the platform to build a dashboard for UCL Museums and collections to display bespoke data about opening times, visitor counts and transport options for the nine separate museums that form the UCL organization responsible for such exhibits. We have also set up various temporary dashboards for bespoke installations within host cities for conferences or other specific shortterm purposes. Due to the modularity of the platform, we can quickly reuse widgets and link them to the overall dashboard that form these temporary pages.

The CityDashboard website also visualises the data contained within the widgets as a secondary view within an interactive map. If a data point contains an item of spatial metadata associated with the sensor, either latitude, longitude or an address, the map view will contain the sensor colour coded to the sensor's type. This allows the user to view the clusters of sensors for which we collect data from within the system. Interestingly, with respect to page views within the website, the map view is under-utilised. Through informal interviewing of users of the platform we understand that a map view may not be the best way to visualise this form of live data. Users report that the data being one extra click away rather than being presented directly to users like the widget view creates a barrier between the user and the data presented. In fact this may be due to users not noticing the link to the Map View on the front page of the website or other user interface factors. This is an interesting finding and further research is needed into this issue at a later date. We are able to create such a system due to the democratisation of data available within cities, chiefly due to the increasing use of open data stores. 


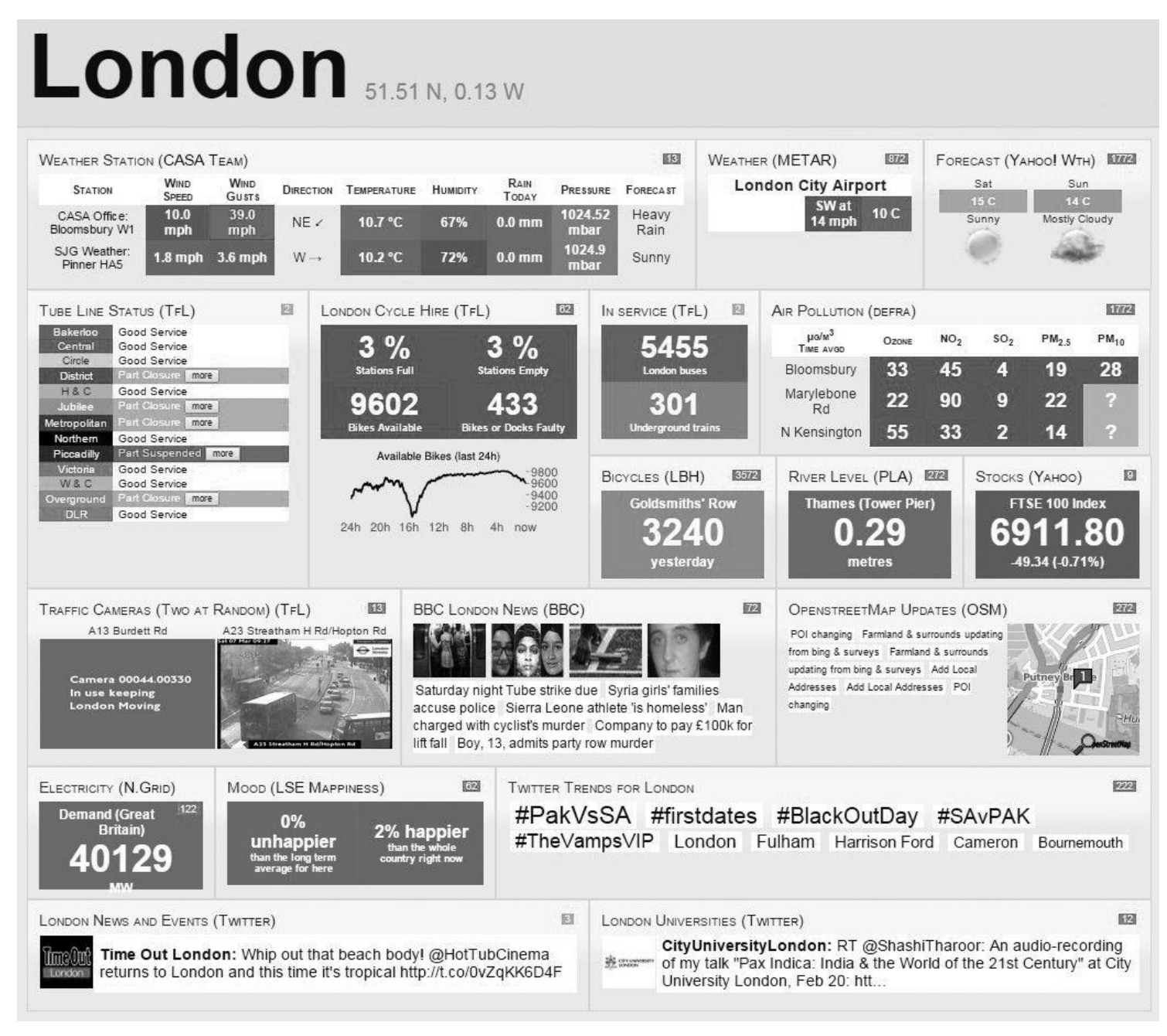

Figure 1: The CityDashboard Website

Urban datastores have become a popular way for some forward-thinking city authorities to share public service information as open data with third party users who they assume constitute their local population in a transparent and straightforward way. This is typically provided through a website showing an index complemented with their metadata, and then either an API or downloadable files, sometimes as a CSV but often in less machine-friendly formats such as Excel or PDF files. Berners-Lee (2006) has proposed a five-star system for rating such data, with 'availability at all' gaining a single star, and up to five stars for linked self-describing data that is in a uniform location and is in a machine readable or open format. Potential uses of a datastore such as this are varied, for example journalists looking to create a story from out of data driven techniques, developers creating applications to help people navigate or interact with the city, or even academic researchers looking to understand city patterns or to develop and test land use and transport models. Different cities release different types of data. The data can vary from static information such as locations of facilities, to near-real-time feeds, for example, live running of public transport services. Datastores serve to collect and aggregate the data, although in some cases they exist as catalogues or directories, rather than containing the data themselves. 
The London Datastore was launched in 2010 by the Greater London Authority, with the personal backing of the city's then high-profile mayor, Boris Johnson. London's governing structure is complicated, with much of the day-to-day running controlled by its 33 boroughs, transport functions being devolved to the agency Transport for London which has its own open data portal, and other functions being handled by the national government which also operates its own datastore. As such, it was a challenge to create the platform. Coleman (2014), who was the champion and architect of the store, found the different degrees of willingness to be open between the various bodies concerned, to be a challenge. The London Datastore acts mainly as a catalogue of data, with a limited API available but little data actually contained within the store itself. As such, it has proved difficult for the catalogue to remain up to date, as source URLs have moved or updates have been published at source, without notifications filtering through to the London Datastore itself.

\section{The API for the CityDashboard}

The project was designed first and foremost as a platform, for use by the accompanying website as a conceptual demonstration but also by other services, both internal to the project and external. As such, the project has an API and the processed and formatted data is made available in a consistent style. The CityDashboard API allows the project to disseminate the metrics to different projects that enhance the overall visualisation by providing data to the these different physical and digital visualisations. The API allows researchers and developers quick access to the metrics without having to maintain and build complex systems to collect the data. The CSV and JSON formats of the API allow for speedy development and integration of the data into other systems without the need for re-implementing complex authentication workflows to collect the data.

As one can imagine with a popular platform, the team get many requests from users and data providers to add data which is important to them to be included within CityDashboard. Within the platform we have stringent rules which have to be met before data can be displayed on the platform. Each widget has to conform to these questions before we consider designing and implementing the widget and finally adding it to the platform. By asking these questions, the team conceptualises the fundamental conditions that make the dashboard a powerful tool for visualising urban data and if all these conditions are met and then passed, we know that we will have an audience for the data without diluting the aesthetics or the interactivity of the dashboard. These questions serve as guidelines and are presented in the following section with a brief discussion about the questions importance.

\section{Is the Data Open?}

This is the most important and overarching question which is not only policy-driven but also technically vital. The CityDashboard serves as a showcase of urban data that is available to third party developers through documented APIs. The data must be open in the sense that the data is reachable from the servers that collect the data from the data sources. If the data is not available on the open web, then it cannot be included in a technical sense within the system. Data providers who do not have an API but provide the data within a website which updates at regular intervals are included within the platform but the team prefer well documented APl's as these types of datasets are maintained and are reliable. The platform 
can scrape data from websites but often datasets of this nature are updated frequently and if the providers introduce changes to these pages then, more often than not, the widget will break for all users of CityDashboard.

\section{Is the Data Updated Frequently?}

Many users report that the biggest draw of the system is that the data is regularly updated, which provides the user with an ever changing live view of the city. If the data inside a widget is not updating regularly, then it becomes static and users have a difficult time discerning between data which is infrequently updated and a broken data source which is not updating. Each widget contains an error state which informs the user when the data is not updating yet these error states are infrequent and many users never see a widget in this state. This guideline ensures that the interface continually evolves with new data that enhances the overall website.

\section{Are Citizens Interested in the Data?}

The data sources have to be interesting and provide some new context about the urban environment around the city' resident citizens and probably visitors. Data which does not fulfill this criteria fundamentally will not appear on the dashboard.

\section{Is the Data Source Available for Multiple Cities?}

Due to the modular system that underpins CityDashboard, data sources which are available for different systems will enhance multiple dashboards. This criteria is not essential for inclusion into the system but a data source which is available for multiple cities will motivate the team to create a widget and include it on all the dashboards within the platform.

\section{Is The Data Morally and Ethically Collected?}

Fundamentally the system is not corporate in nature, nor has it been built for profit or political purposes. It is part of an overarching research project and as such, we decided to undertake an ethical view of the data sourced for inclusion within the system. The data that is visualised within the system should not identify individual persons or groups. The team have taken great care to make sure that the system will never reveal data which could reveal the location of another user. All the data is aggregated at collection - the raw data is disregarded, only the aggregated data is stored and provided to other researchers internally. In the same vein, we do not take data down when it shows any negative undertone of the city, for example disruptions or protests. The only circumstances under which we remove data sources from the platform are when a data provider asks that we stop collecting data due to Terms of Service violations, or the collection servers are put under undue stress on the delivery method of the data providers. While rare, these requests do happen but we use that opportunity to start a dialogue with the providers to give the research team access to the data for ongoing inclusion.

The data sources that power the CityDashboard platform clearly enhance the overall visualisation of live city data. When the data and the visuals works in tandem, the users get the benefit of an ever changing overview of the city. As researchers, the underlying data API can be used to power different applications within the department and other projects which need an archive of data that may be useful to the needs of individual researchers. 


\section{Uses of the CityDashboard API for Visualisation}

Internally to our Centre where we have developed this, we have used the platform to build different physical computing applications which enable us to visualise the data in various ways. Disseminating urban analytics to the public through physical visualisations not only provides a differing experience of that data to the set of users but introduces the data and the issues to a new audience. Two of these types of exhibits using the CityDashboard API are the London Data Table and the iPad Video Wall, two systems that have been used to communicate the data we have collected in unique ways.

\section{The London Data Table}

The London Data Table was built as an exhibit for a one-day bi-annual conference held at CASA entitled Smart Cities, Bridging the Physical and Digital. As the title of the conference hinted at, the exhibition space at the conference explored how we bridge the digital data we produce by our daily actions as well as the physical representation of the data through devices and installations. The London Data Table presents a new way of communicating the city by visualising data on a physical object rather than through the traditional delivery method of video media or through a web visualisation.

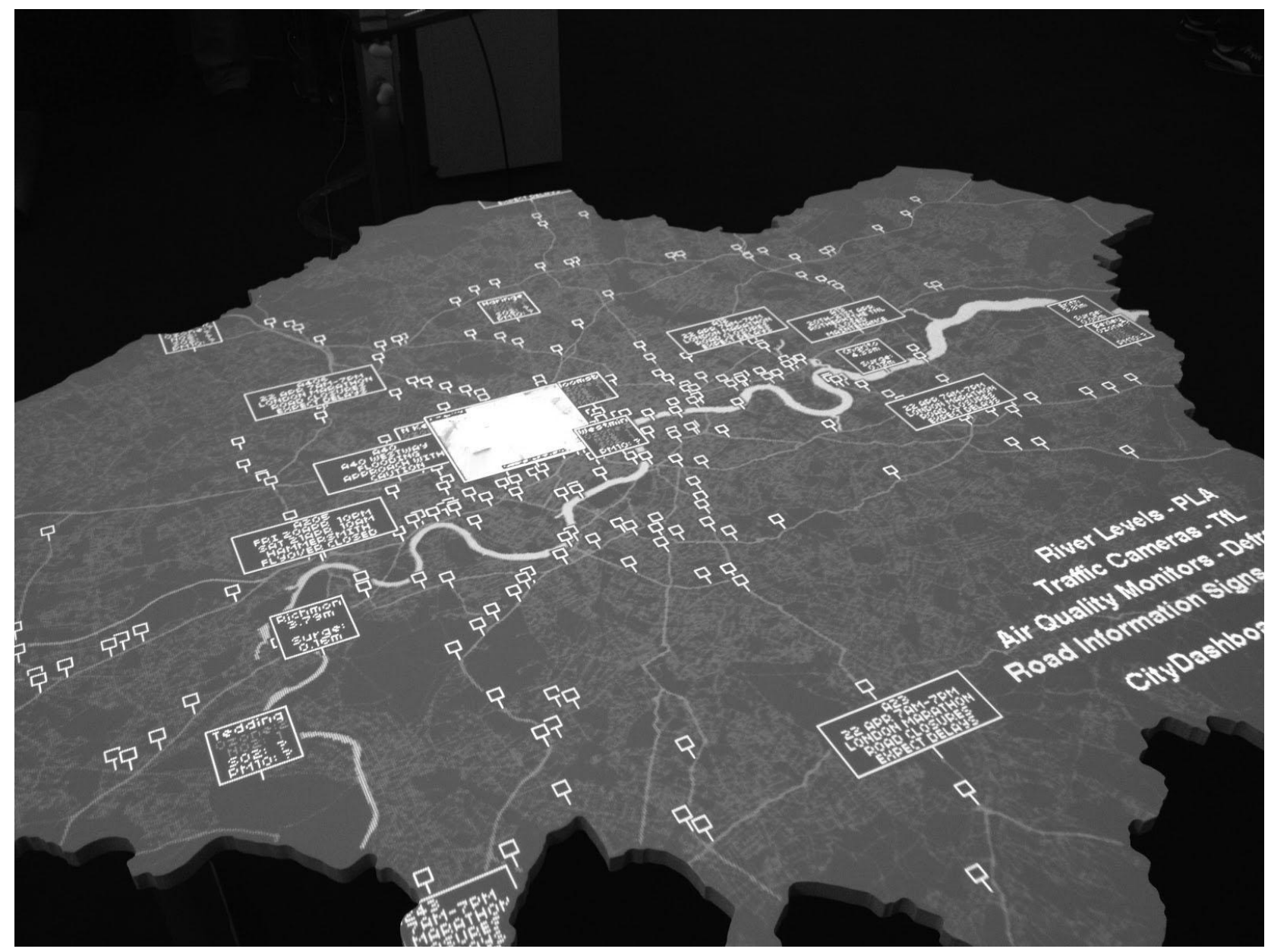

Figure 2: The London Data Table Showing Data from CityDashboard API 
Created in the shape of Greater London, the London Data Table has various visualisations projected onto the surface of the table from overhead, allowing users to have a physical sense of the scale of Greater London as well as viewing compelling visualisations from a bird's-eye perspective which we show in Figure 2. The visualisations that are shown on the table range from live aircraft positions, collected from an ADS receiver mounted at in the university to the sites of various researcher's homes within the Greater London area, all the way to live traffic and bike hire usage as well as public transport visualisations powered by the CityDashboard API.

The visualisations are created in the same fashion for dissemination on the web or TV media (by creating movies or web-based visuals) but are presented via customised basemaps and hardware applications which rotate the visualisation on the table. The table can also be controlled via an iOS or Android application by visitors to the exhibition which displays some background to each scene as well as controls to advance or replay the content on the table.

CityDashboard is represented in the London Data Table through the map visualisation showing the location of each data sensor placed around the city. This view is indistinguishable to aforementioned map view within the CityDashboard website. The only difference between the two views is that the sensors rotate to show a handful of sensors of each type and any one time. Having only a few of the data points open on the map allows the data to refresh and the user to explore the map without having to interact with the table.

The data table allows users to explore the data as a collaborative experience rather than an individualistic experience as in sitting in front of a computer or television. Watching a visualisation movie on a TV or during a lecture is a passive experience where the spectator draws their own views and conclusions about the visualisation. Whereas with the London Data Table, users can stand around the table - in an approximately 270 degree arc, taking into consideration the stand for the overhead projector - and discuss what they experience from the visualisations. This inclusive experience allows for discussion about the amount of data available for London as a whole. Spectators discuss the visualisation and usually reference their home location and discuss local issues raised from the urban data. Specifically, in relation to the CityDashboard project, citizens who view the visualisation on the Table have discussions around the problems facing the city; disruptions, traffic delays stalled trains and signaling failures on the underground network. Air pollution is another topic that is discussed "around the table" especially on days when there is warm weather within London and little wind within the city to move car exhaust particulates around. The table allows this sort of discussion to take place due to number of air quality sensors around London as well as weather stations available within the CityDashboard API which are made available to the table visualisation.

The iPad Video Wall

The iPad Video Wall is a bespoke built exhibit based on the IPad tablet computer, designed to showcase the live and static visualisations of the urban data we collect using the CityDashboard API. The wall was built in 2013 to showcase the CityDashboard platform in the London Mayor's Office in the Greater London Authority (GLA).

The wall contains an array of 12 iPads in a 4 by 3 configuration mounted in a custom-built wooden frame that is lightweight and portable as we show in Figure 3. Each iPad is 
connected via WiFi to a centralised cloud server using a custom iOS application stored on the iPad. The iPads maintain a constant connection to the cloud server so that various commands can be sent to the iPad Wall to change visualisations and provide an interface to carry out various diagnostics on the wall.

In the wall's default configuration, each iPad takes the data from a single widget in CityDashboard and displays the same data on the iPad. The main view shows the live metric which updates in real time and displays some information about the metric. So for example, the Transport for London ( $T f L)$ underground network widget cycles between each of the twelve main lines and displays the status of each line. If a line is other than "Good Service" the the disruption is displayed at the bottom of the page. Each widget has a primary colour to differentiate between each widget and depending on the normalised value of the metric, the background colour of the widget will change. For example for the FTSE 100 widget if the overall value of the market falls during the day the background colour of the widget will change to red. Conversely, if the value increases, the background colour will turn green.

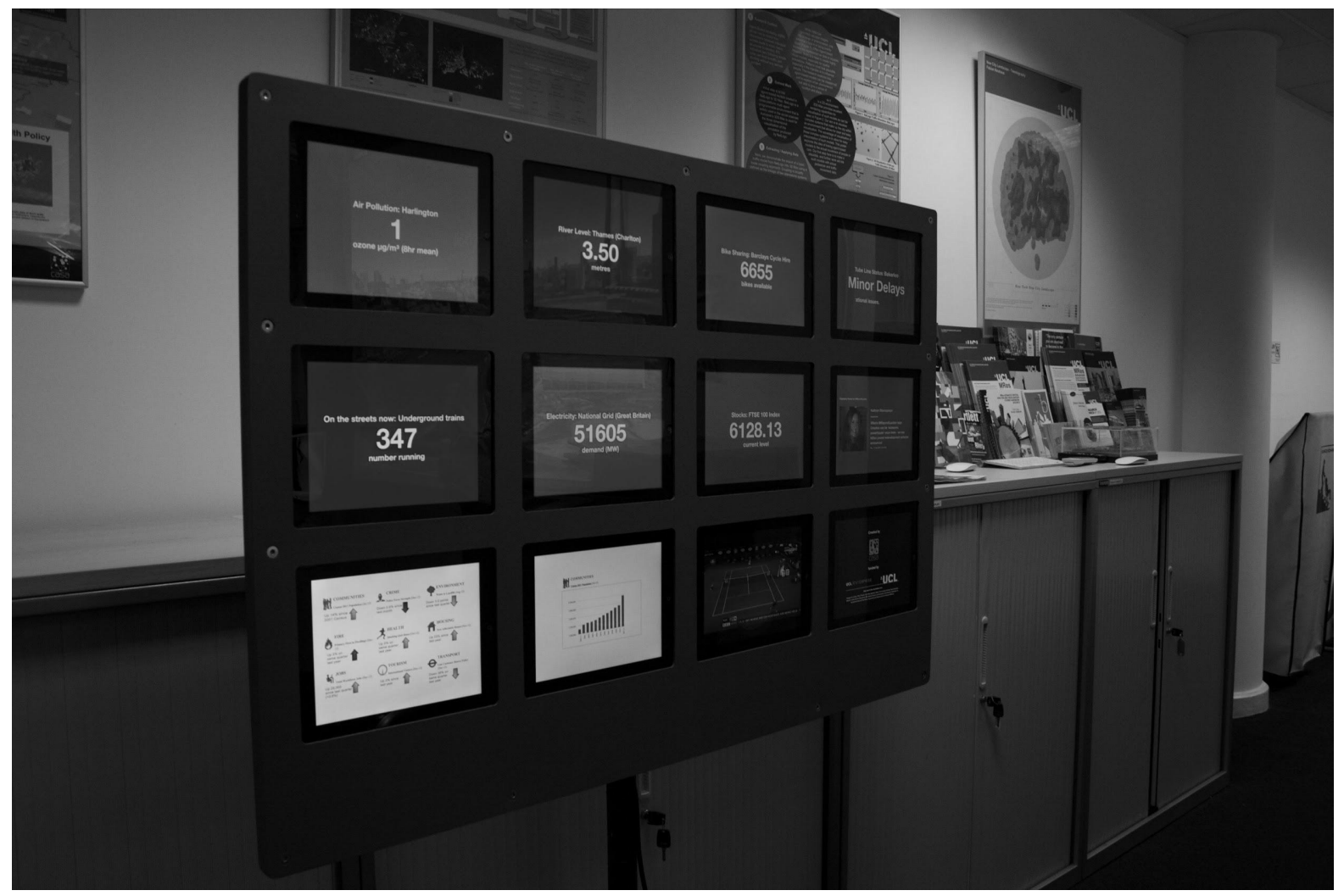

Figure 3: iPad Wall Showing City Dashboard Metrics

When triggered, the iPad Video Wall can change into a presentation mode where each of the iPads shows a segment of an overall bigger rendered movie, providing a window into the visualisation. Each iPad maintains a constant connection with the other iPads in the wall as well as the central server to keep each of the iPads in synchronisation. The movie mode can either be triggered by a user on one of the iPad's by clicking on a movie or by a companion mobile application. 
The advantage of using tablet devices within the wall is that it brings a new interactivity to the urban data displayed. The touch screen interface can be used by the user to interact with the data in a new dimension that is not available on the main CityDashboard website. Each iPad widget has two views, the main live updating single metric, like the number of underground trains in the TfL network, and a secondary view that resides behind the main view. When a user touches the $i P a d$, the view flips over to the secondary view which displays further analysis about the metric. The secondary view usually consists of graphs and other time series data visualisations related to the metric on the main view. This allows the user to drill into and explore the history of the data metrics, a feature that the main CityDashboard website does not show.

\section{The London Periodic Table}

The London Periodic Table, which was created as a more vivid, bespoke version of the CityDashboard website, focusing in a single location in London, specifically the locality surrounding our CASA office. All data in the London Periodic Table is presented in a square, with a single colour, a primary value, and a minimum of supporting information, such as a caption, unit or secondary value. The colour in each square pulsates if the value is unusually low or high. For example, the current observed temperature from the CASA weather station shows in a panel, with a colour ramp from blue (cool) to red (hot). Temperatures above $30^{\circ} \mathrm{C}$, unusual for the central London location that the London Periodic Table is focused on, cause the square to pulse from dark red, to bright red, every few seconds. Since the initial creation of the visualisation, the collection of squares that form the visualisation have been augmented by an additional column of squares for indoor measurements

\section{Prism - at the Victoria \& Albert (VA) Exhibition}

Our last device is called the Prism (Matsuda 2012) which is modelled on a sculpture created by digital/new media artist Keiichi Matsuda and his workshop assistants. Prism presents an alternative view of London by exposing data flows that are generated from everyday interactions from infrastructural data feeds and exchanges. The exhibition is consisted of a 3D object made of a series of connected triangles. A number of projectors, internal to the structure, shone onto each triangular face a visualisation that received data from the CityDashboard API, transforming it into a texture, a sequence of words, or another visual effect. The sculpture was suspended from the ceiling of a gallery in the Victoria and Albert Museum and also protruded through the floor into another gallery below. A nearby staircase allowed visitors to look over the city itself, and contrast their view with the data view presented on Prism. The sculpture was a temporary work, although an accompanying website remains, with the textures that were projected remaining visible.

\section{Extending the Dashboard Narrative}

Through these interactions, it can been seen that using a dashboard as a communication tool is effective for displaying real time summary statistics for cities. However, even though the display is in near real-time, the server side infrastructure of the system still relies on a pull architecture. Specialist collection software running on the server pulls data from the data providers at specific intervals and the data is then displayed within the widgets. The web, as 
a platform for building applications, is moving to real-time communications where information is pushed to clients, or users that are connecting to this type of service.

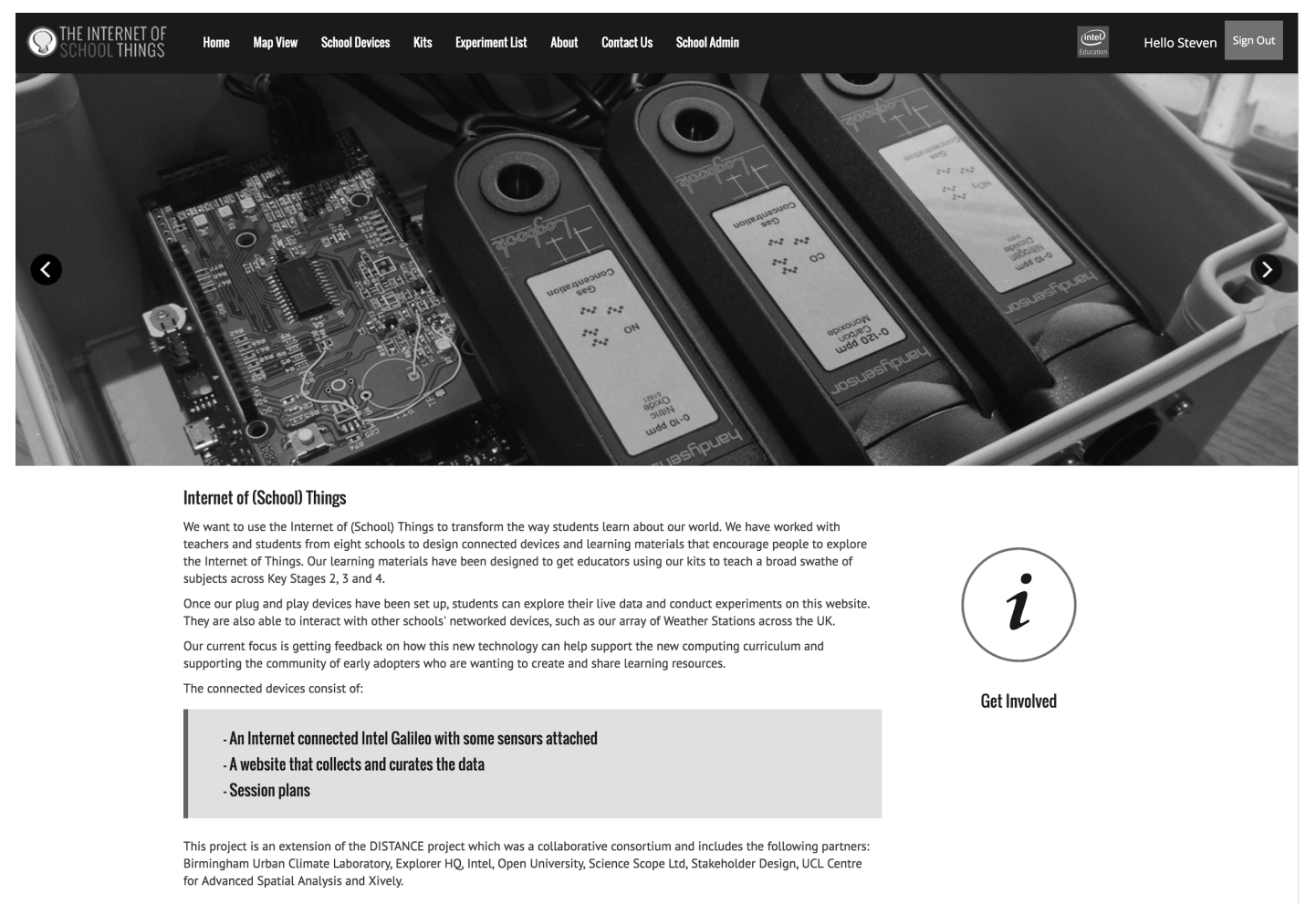

Figure 4: Internet of Schools Project Showing Sensors Placed in Schools

The Internet of School Things is a research project and an educational tool to allow pupils within schools access to real-time data that is being generated around them by sensors within the schools. In collaboration with Intel and ScienceScope, the project is intended to investigate the possibility of using loT sensors and dashboards as teaching and learning tools, by deploying a self-contained suite of sensors in various schools around England.

This suite of sensors which is shown in Figure 4 is comprised of air temperature, ambient noise levels, relative humidity, light levels, and $\mathrm{CO}_{2}$ concentration. As part of this project, the dashboard was adapted to display the sensor data available in the school in which it was deployed, as well as showing comparative metrics from other schools.

We extended the narrative of the Dashboard tool and deployed it within schools which give an additional dimension of interactivity, allowing them to be used as a teaching tool in an innovative manner: a teacher could allow pupils to access the school dashboard, as shown in Figure 5, by unlocking it and supplying a unique URL. The dashboard is then accessible via the Internet, and can be displayed on a variety of devices, from laptops to tablets to mobile phones. The dashboard user interface is made up of tiles which could be added, removed, and re-positioned on demand, reflecting any changes made on the pupils' devices in real time. This was accomplished by using the Firebase real-time data synchronisation library, which instantly propagated changes to all devices in a many-to-many setup using a secure websocket connection. The teacher was thence able to re-lock the dashboard at any time, which placed the dashboard back into a read-only state. 


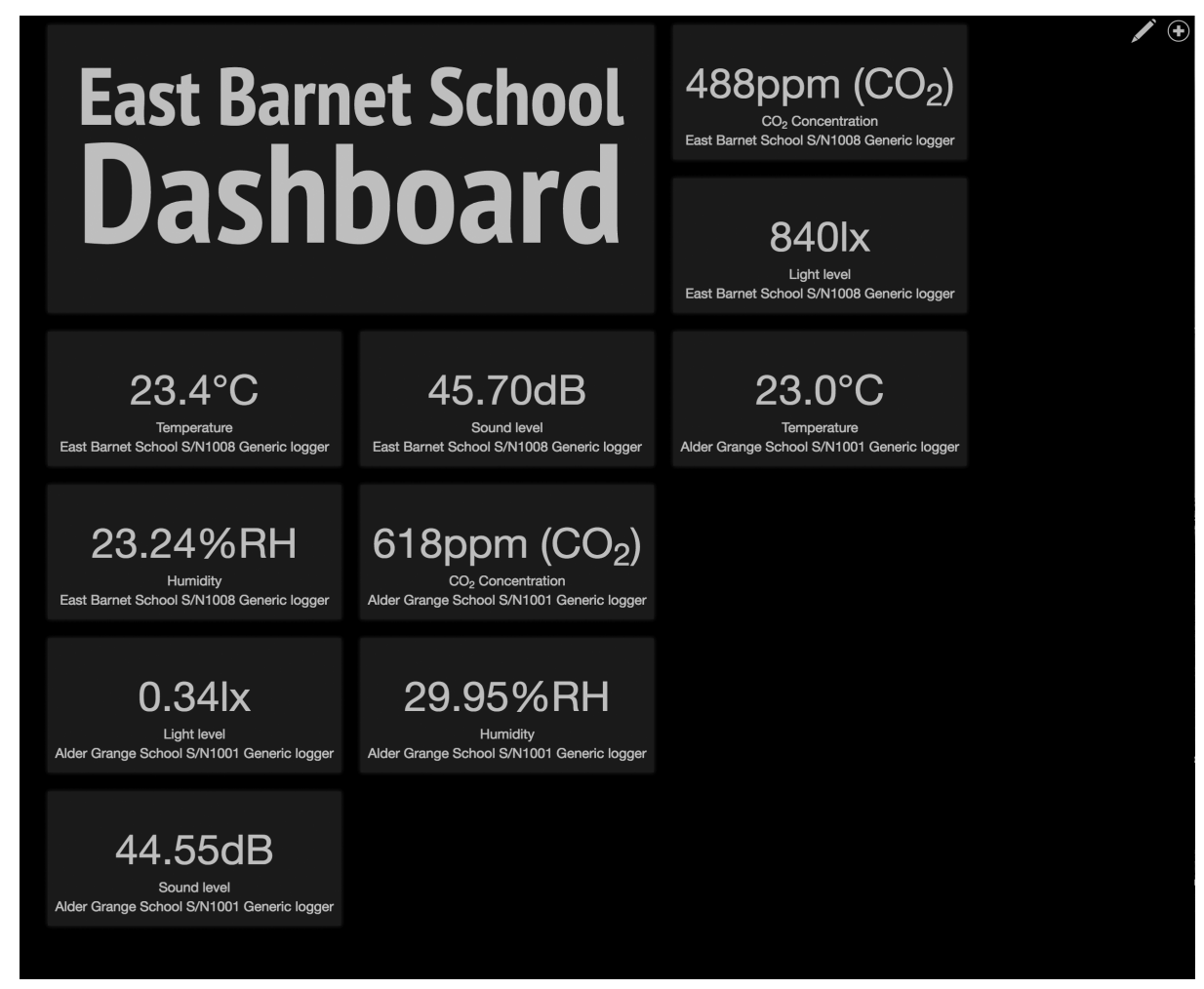

Figure 5: Internet of Schools Dashboard

The real time data from the sensors is pushed to the server and then subsequently pushed to the dashboard as values change within the classroom. When the user loads the website, each widget connects to the relevant datastore for the sensors data, notably Xively. Xively is an Internet of Things platform that updates in real time and allows a centralised sensor data repository to be shared with multiple applications. As each sensor values changes, this will trigger an update to the central server and Xively will send a notification with the new value directly to all users connected to that particular sensor data stream. Using a streaming data retrieval model minimises data transfers to the client as well as load on a third party API. Data is only passed across the network when it has changed and therefore smaller data packets are sent less frequently, unlike a pull data retrieval model, where unchanged data is repeatedly requested and sent to the users' browsers, regardless of a change in the value.

The benefit of using this real time data transfer method is not only to save bandwidth but also to benefit the conversation within the pedagogical environment as an aid to pupils' learning objectives. As environmental values update in real time, then teachers can lead pupils in conversations about changes within the classroom. For example, as more children enter into the room, the temperature will increase as will the $\mathrm{CO}_{2}$ levels. As these values are reflected in real time on the dashboard, the teacher can talk with the class about the effect of the increased $\mathrm{CO}_{2}$ levels on the children or on the plants. These impromptu conversations can be linked to the science curriculum in various ways through lesson plans and through guided, teacher-led science experiments.

The use of interactive, two-way customisable dashboards has proved to be a successful tool for demonstrating the loT sensor suite; the live data, and the ability of pupils to customise 
and rearrange it, seeing changes instantly reflected across the classroom, was seen as exciting and compelling by pupils, and useful and engaging by teachers.

\section{Conclusions}

In this paper we have discussed the collection and visualisation of real-time urban datasets to the cities through the medium of interactive dashboards and novel interaction techniques. These dashboards are providing an interface to view a "tangible, or in some way comprehensible, hard-to-grasp aspect of urban quality-of-life" (Mattern, 2015). Urban informatics remains an emerging field. It is one which is seeing many ideas and a number of prototype implementations coming to fruition - but one in which there is a considerable research and visualisation potential still to be explored and exploited. The great rise of socalled 'big data' adds challenges of its own - not least in its sheer size - but also increases the richness, frequency and quality of data available to be visualised and explored when trying to understand our cities, inform decision makers and make them better places to experience and live in. The tools we have introduced here we consider are part of the set of many ideas that will see smart cities and smarter citizens come to fruition,

\section{References}

Batty, M. and Hudson-Smith, A. (2005) Urban Simulacra: From Real to Virtual Cities, Back and Beyond' Architectural Design, 75 (6), 42-47.

Berners-Lee, T. (2006) Linked Data. Available at http://www.w3.org/Designlssues/LinkedData.html

Coleman, E. (2013) Open Data and Reflections from the London Datastore. WEBIST, IS-5.

CUSP (2014) Urban Informatics, Center for Urban Science and Progress. Available at http://cusp.nyu.edu/urban-informatics/

Geddes, P. (1915) Cities in Evolution. Williams and Norgate, 1949 Edition, London.

Lafler, Kirk Paul (2015), Dynamic Dashboards Using SAS®, Proceedings of the 2015 SAS Global Forum (SGF) Conference, Software Intelligence Corporation, Spring Valley, CA, USA. http://support.sas.com/resources/papers/proceedings15/3487-2015.pdf

Matsuda, K. (2012) Prism. Available at http://keiichimatsuda.com/prism/

Mattern, S. (2015) Mission Control: A History of the Urban Dashboard. Available at https://placesjournal.org/article/mission-control-a-history-of-the-urban-dashboard 